\title{
Outcomes of orthopedic surgery in a cohort of 49 patients with X-linked hypophosphatemic rickets (XLHR)
}

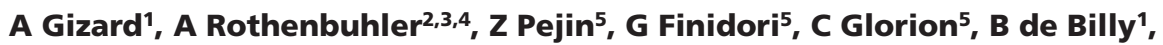 \\ A Linglart ${ }^{2,3,4,6}$ and $\mathbf{P}$ Wicart ${ }^{3,5}$ \\ ${ }^{1}$ Department of Pediatric Orthopedic Surgery, Besançon University Hospital, Paris, France \\ ${ }^{2}$ APHP, Department of Pediatric Endocrinology, Bicêtre Paris Sud, Le Kremlin Bicêtre, France \\ ${ }^{3}$ Reference Center for Rare Disorders of Calcium and Phosphate Metabolism, Le Kremlin Bicêtre, France \\ ${ }^{4}$ Plateforme d'Expertise Paris Sud Maladies Rares and Filière OSCAR, Bicêtre Paris Sud, Le Kremlin Bicêtre, France \\ ${ }^{5}$ APHP, Department of Pediatric Orthopedic Surgery, Necker Hospital, Paris, France \\ ${ }^{6}$ INSERM U1169, Hôpital Bicêtre, Le Kremlin Bicêtre, et Université Paris-Saclay, Le Kremlin Bicêtre, France
}

\author{
Correspondence \\ should be addressed \\ to A Linglart or P Wicart \\ Email \\ agnes.linglart@aphp.fr or \\ p.wicart@aphp.fr
}

\begin{abstract}
Background: X-linked hypophosphatemic rickets (XLHR) is due to mutations in PHEX leading to unregulated production of FGF23 and hypophosphatemia. XLHR is characterized by leg bowing of variable severity. Phosphate supplements and oral vitamin analogs, partially or, in some cases, fully restore the limb straightness. Surgery is the alternative for severe or residual limb deformities.

Objective: To retrospectively assess the results of surgical limb correction in XLHR (osteotomies and bone alignment except for 3 transient hemiepiphysiodesis).

Methods: We analyzed the incidence of recurrence and post-surgical complications in 49 XLHR patients $(29 \mathrm{~F}, 20 \mathrm{M})$ (mean age at diagnosis 6.0 years $( \pm 7.1)$ ).

Results: At first surgery, the mean age was 13.4 years $( \pm 5.0)$. Recurrence was observed in $14 / 49(29 \%)$ patients. The number of additional operations significantly decreased with age (2.0 $( \pm 0.9), 1.7( \pm 1.0)$ and $1.2( \pm 0.4)$ in children $<11$ years, between 11 and 15 , and $>15$ years; $P<0.001)$. Incidence of recurrence seemed to be lower in patients with good metabolic control of the rickets ( $25 \%$ vs $33 \%$ ). Complications were observed in $57 \%$ of patients.

Conclusion: We report a large series of surgical procedures in XLHR. Our results confirm that phosphate supplements and vitamin D analog therapy is the first line of treatment to correct leg bowing. Surgery before puberty is associated with a high risk of recurrence of the limb deformity. Such procedures should only be recommended, following multidisciplinary discussions, in patients with severe distortion leading to mechanical joint and ligament complications, or for residual deformities once growth plates have fused.
\end{abstract}

\author{
Key Words \\ - X-linked \\ hypophosphatemic rickets \\ - osteotomy \\ $\checkmark$ PHEX \\ - hemiepiphysiodesis \\ - genu varum \\ genu valgum
}

\section{Introduction}

$\mathrm{X}$-linked hypophosphatemic rickets (XLHR) is the most frequent cause of genetic and heritable rickets. It is caused by mutations damaging the function of the endopeptidase encoded by the PHEX gene located on the X chromosome (1). The PHEX protein is expressed in the mineralized tissues, i.e. the bone matrix and the teeth. When PHEX is impaired, fibroblast growth factor 23 (FGF23) is produced in excess, leading to renal phosphate wasting and insufficient production of $1,25(\mathrm{OH}) 2$ vitamin $\mathrm{D}$, thereby altering the function of target organs including the bone



This work is licensed under a Creative Commons Attribution-NonCommercial 4.0 International License. 
and the growth plate (2). Children affected with XLHR present with rickets, osteomalacia, enlarged joints, bone pain, growth retardation, abnormal mineralization of the teeth and leg bowing (3).

The last of these usually appears when the child starts to walk; it may be of variable importance and no predictive factors have been identified (Fig. 1). In most patients, the deformity involves the frontal plan leading to genu varum or genu valgum or the combination of both (Figs 1 and 2). There is often concomitant bone twisting $(4,5,6,7,8)$.

The current treatment for XLHR comprises phosphate supplements in multiple daily doses and active vitamin D analogs in order to heal the rickets and the osteomalacia, restore the growth velocity and straighten the legs. To achieve these objectives, the treatment requires a rigorous follow-up and regular adjustments based on weight, and other growth measures, and biochemical parameters. In addition, early initiation of the medical treatment has been associated with better outcomes $(3,8,9,10,11)$.

Orthopedic corrective surgery is indicated if there is marked leg bowing, with immediate functional consequences or long-term threats to the joints. Usually, the bone deformities are corrected through one or multiple osteotomies associated with internal or external osteosynthesis $(4,12,13,14,15)$. Some surgeons progressively correct the angles by transient hemiepiphysiodesis $(4,16,17)$. Recurrence of the bowing has frequently been reported $(4,6,7,15,16,17,18)$. As for every surgery, unexpected consequences have been described, these including pseudarthrosis at the site of the osteotomy $(7,14,17,18,19)$ and pathological fractures $(6,15,17)$.
The main aims of our retrospective multicenter study were 1- to evaluate the long-term results of the orthopedic surgery performed during childhood in XLHR patients and 2- to identify factors predictive of the course.

\section{Methods}

Patients were included in the study if: they had a clinical and biochemical profile compatible with the diagnosis of XLHR, when possible, the diagnosis being confirmed through a genetic analysis; and they underwent at least one surgery of the lower limbs between 1974 and 2014 in one of the 4 centers. The first surgery occurred in 11 patients between 1974 and 1983, in 10 patients between 1984 and 1993, in 8 patients between 1994 and 2003 and in 20 patients between 2004 and 2014 .

We included all patients affected with XLHR who underwent at least one orthopedic surgery in Necker hospital, Paris and in the Centre Hospitalier Universitaire, Besançon, France. Patients were identified through the Reference Center of Rare Diseases of Calcium and Phosphate Metabolism that encompasses both surgical departments.

We collected data related to age at diagnosis, bone phenotype, surgery, active vitamin D and phosphate supplement therapies and alkaline phosphatase level at the last visit.

The metabolic control of the disease was assessed by the bone deformity, i.e. the distance measured between the knees or the ankles, and the alkaline phosphatase level at the last visit. Unfortunately, too many data were missing to include this parameter in the analysis.
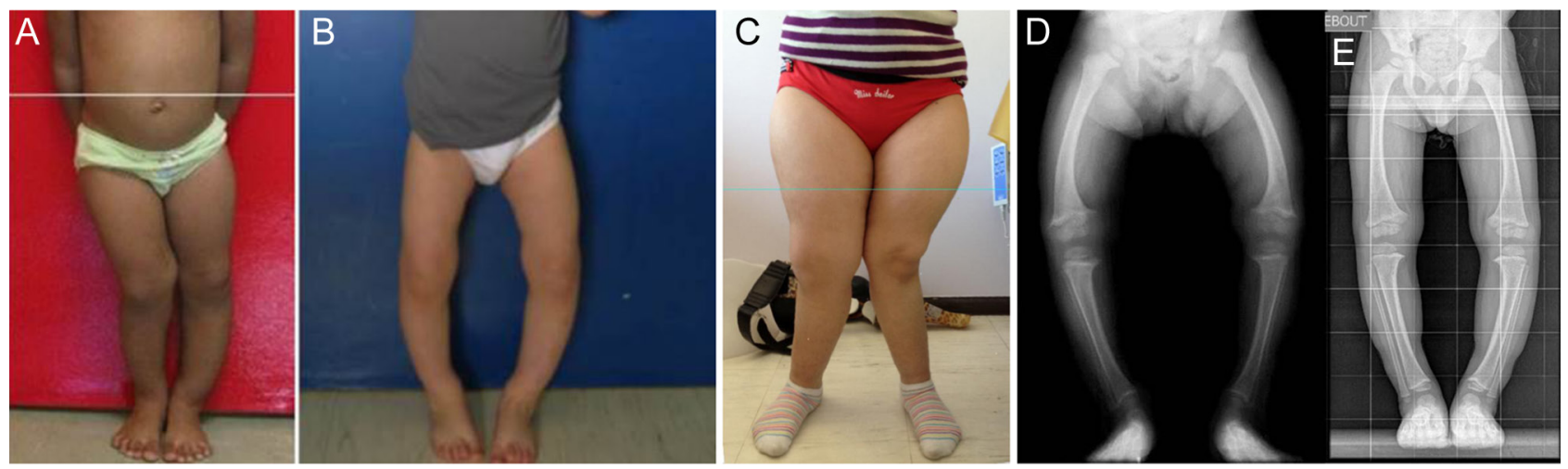

Figure 1

(A, B and C) Leg bowing in 3 patients affected with XLHR (mutation in PHEX). Note the genu varum and genu valgum in (A), genu varum in (B) and genu valgum in (C). (D and E) X-rays of the lower limbs in 2 XLHR children at the time of diagnosis at the age of 4 years (D) and 3 years (E).

http://www.endocrineconnections.org $\quad$ C 2017 The authors
$\begin{array}{ll}\text { DOI: } 10.1530 / \text { EC-17-0154 Published by Bioscientifica Ltd }\end{array}$

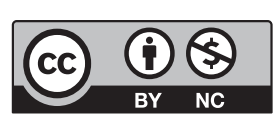

This work is licensed under a Creative Commons Attribution-NonCommercial 4.0 International License. 


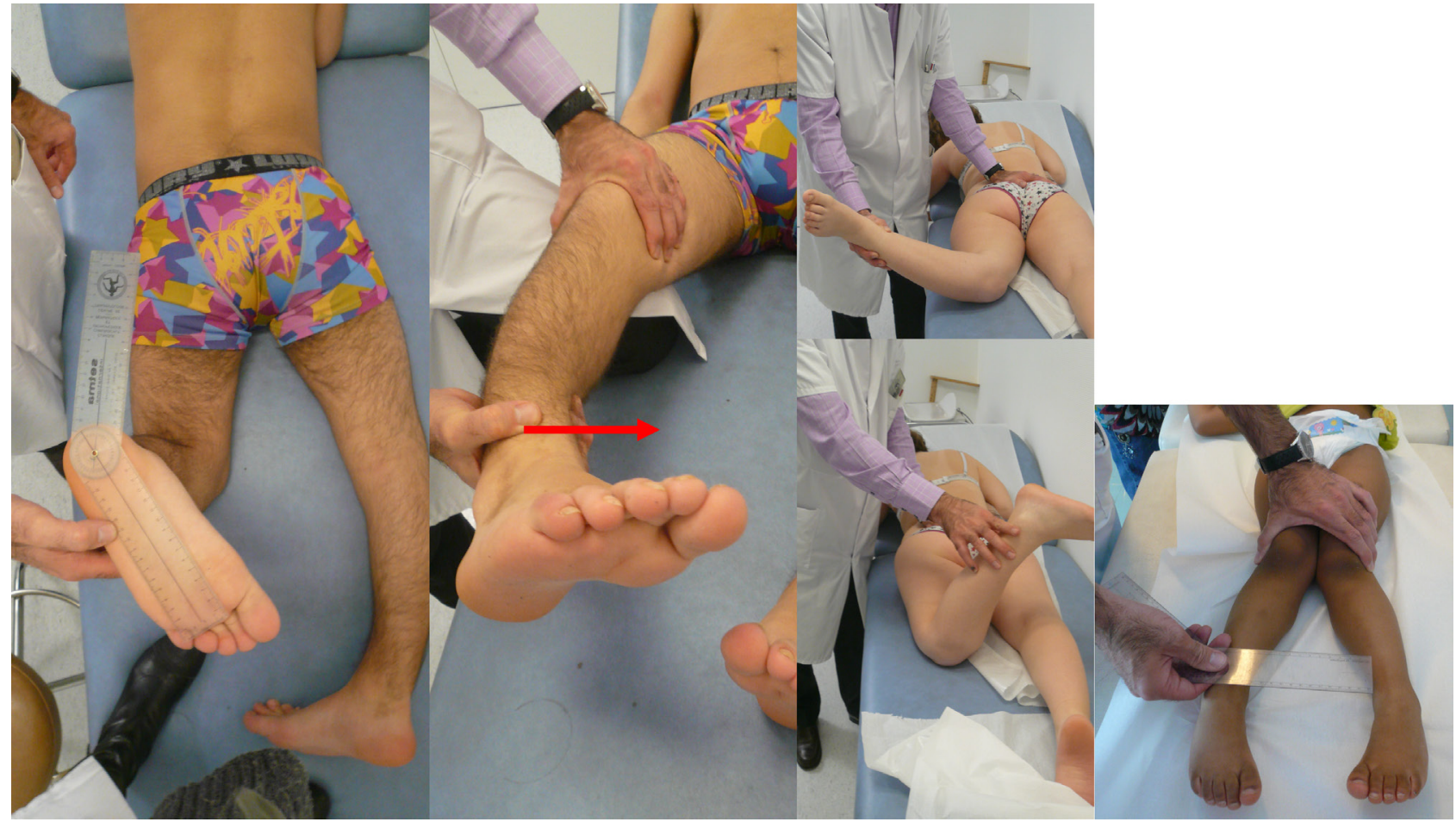

Figure 2

Bone deformity and torsion of the legs shown during the examination of the patients.

The local institutional review board of the Center of Reference of Rare Diseases of Calcium and Phosphate Metabolism approved the study. All patients and parents consented to this retrospective study. Written informed consent has been obtained from each patient or subject after full explanation of the purpose and nature of all procedures used.

Differences between groups were assessed using unpaired nonparametric tests; for qualitative data, comparisons were performed with a $\chi^{2}$ test. The significance threshold was set at $P \leq 0.05$. Statistical analyses were carried out using GraphPad PRISM (v6). All values are shown as mean \pm s.D.

\section{Results}

\section{Patients}

Forty-nine patients (20 girls and 29 boys) affected with XLHR were included in this four-center retrospective study. At diagnosis, the mean age was 6.0 years $( \pm 7.1)$ (Table 1 ). A total of 32 (out of the 49 ) patients (65\%) presented with a genu varum at the time of the first surgery; the mean distance measured between the knees while lying flat (hereafter called the intercondylar distance) was $11.1 \mathrm{~cm}$
( \pm 6.4$)$. The remaining patients $(35 \%)$ presented with a genu valgum; the mean distance measured between the ankles (hereafter called the intermalleolar distance) was $14.3 \mathrm{~cm}( \pm 5.7)$ (Fig. 2). The leg deformity was asymmetric in $20 / 49$ patients. Six and two patients presented with a knee hyperlaxity and a patella sub-dislocation at the time of the first surgery, respectively.

Prior to surgery, most of the patients (42/49) received the current standard therapy for XLHR, i.e. oral vitamin $\mathrm{D}$ analogs and phosphate supplements. Seven patients were not appropriately treated for several reasons, i- the diagnosis had not been made, ii- the patients were operated on before 1980 (active vitamin D only having become available in France in 1975) or iii- the active vitamin D was not available in their country of origin. Only around half of the patients (29/49) had a value of alkaline phosphatase within the normal or close to the normal range at the time of their last assessment.

We divided the 49 patients into 3 groups based on age at first surgery. Group A comprised 13 patients (26\%) who had their first surgery before puberty, i.e. $<11$ years. Group B comprised 20 patients (41\%) who had their first surgery during puberty, i.e. $>11$ years and $<15$ years. Group C comprised 16 patients (33\%) who had their first surgery after puberty and after the end of growth, i.e. $>15$ years. In

$$
\begin{array}{lr}
\text { http://www.endocrineconnections.org } & \odot 2017 \text { The authors } \\
\text { DOI: } 10.1530 / \text { EC-17-0154 } & \text { Published by Bioscientifica Ltd }
\end{array}
$$


Table 1 Characteristics of the patients.

Number of patients (\%)
Patients with an identified mutation in the
PHEX gene $(N)$
Patients with alkaline phosphatase levels
within the normal range at the last visit $(N)$
Age at diagnosis (years)
Sex (M/F)
Age at 1st surgery (years $( \pm$ S.D.); min; max)
Age at last visit (years $( \pm$ S.D.))
$Z$-Score of height at last visit (mean ( \pm s.D. $)$ )*

\begin{tabular}{c}
$\begin{array}{c}\text { Group A 1st surgery } \\
<\mathbf{1 1} \text { years }\end{array}$ \\
\hline $13(26)$ \\
7 \\
5 \\
$5.0( \pm 4.9)$ \\
$5 / 8$ \\
$7.7( \pm 2.7) ; 3.1 ; 10.8$ \\
$26.0( \pm 13.1)$ \\
$-3.3( \pm 2.1)$ \\
\hline
\end{tabular}
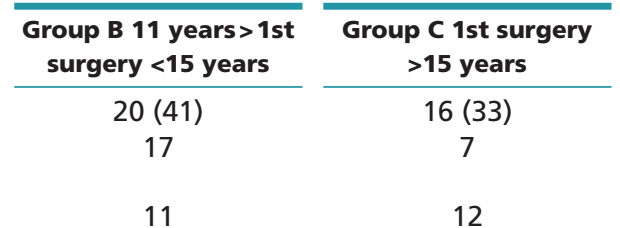

Comparison of groups

$\begin{array}{cc}6.3( \pm 8.3) & 6.6( \pm 8.3) \\ 8 / 12 & 9 / 7 \\ 13.2( \pm 1.3) ; 11.0 ; 14.9 & 18.3( \pm 4.6) ; 15.1 ; 31.5 \\ 21.6( \pm 9.7) & 23.1( \pm 6.8) \\ -2.3( \pm 1.4) & -2.6( \pm 1.8)\end{array}$

NS, $P=0.967$

$P<0.0001$

NS, $P=0.388$

NS, $P=0.608$

*1, 4 and 1 patients in group A, B and C received growth hormone for their short stature, respectively; NS for not significant.

addition, in each group, we particularly closely examined the cases of patients with uncontrolled metabolic rickets versus those with controlled disease.

\section{Surgery}

Considering the entire cohort, the mean age at first surgery was 13.4 years $( \pm 5.1)$ (Fig. 3). Overall, $46 / 49$ patients underwent an osteotomy with correction and fixation to restore the alignment of the bones (Fig. 4A and B). The correction of the deformity was either progressive or acute using external or internal fixation. Overall, 22 (45\%) and 36 (74\%) patients underwent a femoral and a tibial osteotomy, respectively. In 16 patients, both osteotomies were done concomitantly. The osteotomies achieved an effective correction of lower limb deformity with post-operative inter-condylar and malleolar distances of less than $2 \mathrm{~cm}$. Only three patients had a transient unilateral hemiepiphysiodesis, a technique based on the principle of guiding the growth. This procedure did not provide significant limb axis correction.

We were able to retrieve follow-up data on the patients for an average period of 9.4 years after their first surgery.

Overall, 14 patients had recurrence after their first surgery, in a similar manner to the initial bone deformity in all cases (9 in varus and 5 in valgum). The recurrent distortion occurred in the close vicinity of the osteotomy, i.e. proximal in the case of femoral osteotomy and distal in the case of tibial osteotomy (Fig. 4C). However, in



Figure 3

Flow chart of the surgical operations and course over time. Patients are divided into 3 groups (, , B and C) according to their age at first surgery. The number of patients with controlled or non-controlled rickets is shown. Overall, the mean number of operations per patient is shown in the bottom panel. Statistically significant differences between the three groups are indicated $(*)$. 

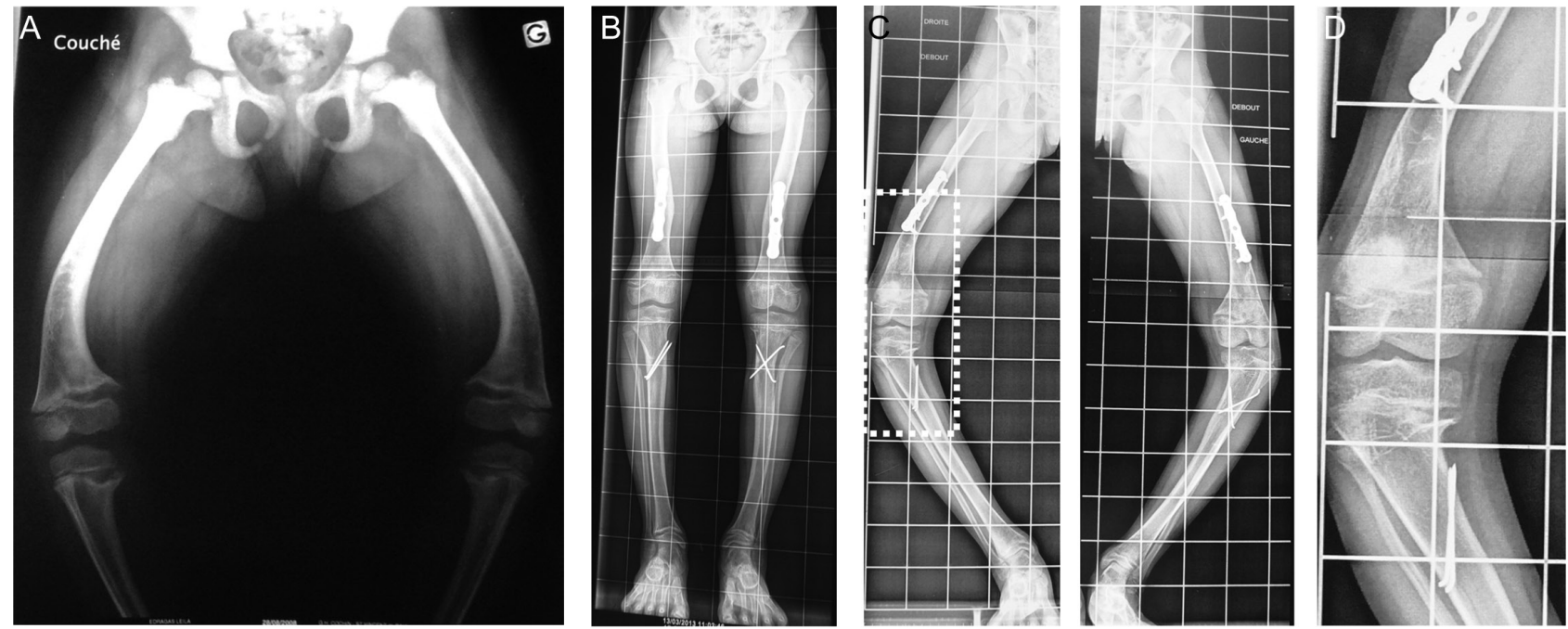

Figure 4

Post-osteotomy changes and complications in a patient with severe rickets due to XLHR. (A) X-rays of the legs prior to the first surgery (age 7.9 years). (B) Correction of the leg deformity immediately after the surgery. (C) Recurrence of the varum below the femoral osteotomy (age 9.5 years).

(D) Enlarged picture of the femoral and tibial physis (framed in (C)) showing the medial epiphysiodesis although the surgery was performed at distance from the physis.

three patients, the recurrence of the bowing was caused by a bilateral spontaneous asymmetric epiphysiodesis occurring at a distance from the initial surgery site. In two patients, the epiphysiodesis was located at the medial compartment from the proximal tibial metaphysis (Fig. 4D). In one patient, it affected, the medial compartment of the distal tibial physis.

The rate of recurrence appeared lower, although not significantly, when patients were older at first surgery (46, 25 and 19\% in groups A, B and C, respectively) (Fig. 3). In addition, the number of iterative surgical procedures was inversely correlated with age at first surgery $(2.0( \pm 0.9), 1.7$ $( \pm 1.0)$ and $1.2( \pm 0.4)$ in groups $A, B$ and $C$, respectively; $P=0.0221$ ). Medical control of the rickets assessed by the ALP level at the last follow-up only showed a tendency to favor a better outcome (25 and 33\% of recurrence in patients with a metabolic controlled vs uncontrolled rickets, respectively). Patients with an intercondylar distance below $12 \mathrm{~cm}$ (median of intercondylar distances in this series) had similar outcomes (recurrence, number of surgeries and complications) than those with an intercondylar distance superior to $12 \mathrm{~cm}$. In two patients, the surgical hemiepiphysiodesis, performed at the age of 11.5 and 12 years, did not improve the leg shape at all. Both patients underwent additional surgeries thereafter to correct their leg deformities. In one patient, the improvement was described as moderate at 6 months after the surgery. The patient did not go through any additional surgical intervention.
C) 2017 The authors Published by Bioscientifica Ltd
In average, patients who underwent their first surgery between 1974 and $1983(n=11)$, when compared to patients operated between 2004 and $2014 \quad(n=20)$, were diagnosed later for XLHR, showed more severe bone deformities, had their first leg surgery earlier, went through a larger number of limb surgeries, experienced a greater rate of recurrence and complications and had a shorter final height (Supplementary Table 1, see section on supplementary data given at the end of this article).

Twenty-eight (57\%) patients experienced at least one surgery-related complication, including recurrence (18/49 if we exclude the recurrences), leading to an additional operation in $25 \%$ of cases. The mean number of complications in these 28 patients was $1.7( \pm 0.5)$. Neither age at first surgery nor medical control of the rickets was associated with the occurrence of these complications. The rate of complications was 25, 14, 17 and 100\% after one, two, three or more operations, respectively. Apart from recurrences, the reported complications were pseudoarthrosis, fractures, infection, ossification around a pin, early arthrosis and hypoesthesia in the area of the inferior tibial nerve.

\section{Discussion}

Our objective herein is to report our experience in the limb surgery of patients affected with XLHR to identify factors predictive of the outcomes and highlight the specific characteristics of this type of surgery in the context of XLHR.

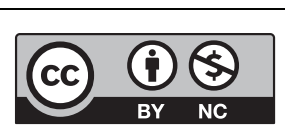

This work is licensed under a Creative Commons Attribution-NonCommercial 4.0 International License. 
To our knowledge, this cohort of patients with XLHR who have undergone surgery is among the largest reported so far $(5,6,7,8,12,13,14,15,18,19)$. It would be reasonable to expect that patients with this condition who require surgery to be the most severely affected. In line with this, the mean final height of the entire cohort is $-2.7( \pm 1.7)$, less than the previously reported mean adult height in XLHR $(10,11,20,21,22)$. Noteworthy, the mean age at diagnosis of this cohort is higher than what is usually reported in series of XLHR patients. This may be due to the selection of patients restricted to those who required surgery. Eight out of 49 patients were diagnosed after the age of 10 years, including 4 patients who underwent surgeries before the diagnosis of the disease was made.

One major finding of this study is the high rate of recurrence after the first surgery (29\%), confirming the reports in smaller case series $(27 \%$ in $(17,18,19), 25 \%$ in (7) and $90 \%$ in $(4,6,15))$. Overall, the risk of recurrence appears to be related with young age at first surgery, as demonstrated by the inverse association between age at first surgery and the number of operations, and the decreasing rate of recurrence in groups $\mathrm{A}, \mathrm{B}$ and $\mathrm{C}$. As above, this result is in accordance with previous published reports $(4,5,6,17)$. For instance, in a series of 10 XLHR patients, Petje and coworkers described $90 \%$ of recurrences after first surgery (average age 8 years and 10 months) and $60 \%$ after second surgery, leading to a mean of 3.7 procedures per patient (6).

In our series of XLHR patients, the control of the rickets, assessed by the level of alkaline phosphatase at the last follow-up visit, was not associated with the rate of recurrence or with the complications. Our analysis may have been limited because we could not assess the metabolic control at the time of the 1st surgery. In contrast, Rubinovitch and coworkers reported a strong effect of the biochemical control of the disease in preventing recurrences in a series of 10 XLHR children. Notably, the assessment of the metabolic control of the disease in their publication was more stringent than ours, as it included $\mathrm{X}$-rays and bone biopsy patterns, alkaline phosphatase level, growth rate and progression of the bone deformity (18). Other groups have described similar experiences $(8,19)$. XLHR is a metabolic disorder associated with increased circulating FGF23 levels, and hence, phosphate wasting. Ineluctably, in the absence of medical treatment, the bone is abnormally mineralized and the growth plate is disorganized because of the decreased apoptosis of the chondrocytes. Therefore, following surgery, open physes are prone to rickets, and hence bone deformities if the medical therapy is not sufficient. The findings of our study might have been limited by $i$ - the assessment of the metabolic control, ii- the size of the sample and iii- the retrospective analysis of the data.

We report here, for the first time, three cases of spontaneous post-surgical epiphysiodesis, likely reflecting the severity of the disease affecting the physis in the absence of adequate medical therapy. In two studies, premature fusion of the pathological growth plates was suspected. Rohmiller and coworkers proposed that the short stature of patients that had been operated on might be due either to the surgical procedure itself or to the severity of the disease (7). In a large series of 47 patients, Popkov and coworkers observed that some patients who underwent osteotomies and internal nailing had newly formed deformities in the distal femoral and proximal tibial metaphysis (15).

Hemiepiphysiodesis is an alternative procedure to osteotomy, which could limit the bone deformity in young patients (16). However, in accordance with Eyres and coworkers, we observed poor corrections and a high rate of recurrence associated with this technique (14), limiting its use in daily practice.

To limit the number of surgical procedures per patient, and hence the number of complications, based on our results and a critical analysis of the literature, we recommend close monitoring of patients in the context of multidisciplinary care involving the medical team responsible for adjustment of the phosphate supplements and vitamin $\mathrm{D}$ analogs and the radiologist responsible for imaging the bones and assessing the degree of the leg deformities. As the current medical treatment allows a complete or a partial restoration of the leg shape $(8,19)$, it is of utmost importance to diagnose the disease as early as possible, start the medical treatment as soon as the diagnosis is established and obtain an optimal metabolic control of the rickets, i.e. lack of bone pain, growth velocity normal for the age, normal or near-normal alkaline phosphatase levels and absence of signs of active rickets on X-rays of the physes. In XLHR patients, it might be beneficial to delay the surgery to closer to the end of the growth, to obtain this metabolic control of the disease and perform only one corrective surgery. However, some patients may require an early operation in the case of joint complications such as femoro-tibial instability, or patella sub-dislocation, or if there is an increased risk of mid-term or long-term consequences for the joints (for instance, early arthrosis).

Surgical correction of leg bowing is a difficult task in patients affected with XLHR. The incidence of recurrence 
is high, even in expert hands. In addition, complications such as spontaneous fusion of the pathological growth plate occur distant from the surgical site. Overall, the indication for surgical procedures in these patients should be considered bearing in mind the age of the child, metabolic control of the rickets, risk of long-term complications, risk of recurrence and potential iterative operations. Surgery before puberty should be avoided as it seems to be associated with a higher rate of recurrence. Our hope is that novel therapies such as antiFGF23 will prevent and/or heal the orthopedic complications encountered by XLHR children.

\section{Supplementary data}

This is linked to the online version of the paper at http://dx.doi.org/10.1530/ EC-17-0154.

\section{Declaration of interest}

A Linglart has received honoraria and travel fees from Ultragenyx and Kyowakirin. A Rothenbuhler has received honoraria from Kyowakirin.

\section{Funding}

This work was supported by grants from the APHP (Plateforme d'Expertise Maladies Rares Paris Sud) to A L and A R and the SOFOP (Société Française d'Orthopédie Pédiatrique) to A G.

\section{Author contribution statement}

The work described has not been published previously, and its publication is approved by all authors. All authors have made substantial contributions to the following: the conception and design of the study ( $A G, A L, P W$ ), acquisition of data ( $A G, A R, Z P, G F, C G, B d B, A L, P W$ ), analysis and interpretation of data ( $A, G, A R, A L, P W$ ) and drafting the article ( $A G$, $A L, P W)$, and all have given final approval of the version to be submitted (A G, A R, Z P, G F, C G, B d B, A L, P W).

\section{Acknowledgements}

The authors are very grateful to the patients and their families for their contribution to the study.

\section{References}

1 Bergwitz C \& Jüppner H. FGF23 and syndromes of abnormal renal phosphate handling. Advances in Experimental Medicine and Biology 2012728 41-64. (doi:10.1007/978-1-4614-0887-1_3)

2 Feng JQ, Clinkenbeard EL, Yuan B, White KE \& Drezner MK. Osteocyte regulation of phosphate homeostasis and bone mineralization underlies the pathophysiology of the heritable disorders of rickets and osteomalacia. Bone $2013 \mathbf{5 4} 213-221$. (doi:10.1016/j.bone.2013.01.046)

3 Linglart A, Biosse-Duplan M, Briot K, Chaussain C, Esterle L, Guillaume-Czitrom S, Kamenicky P, Nevoux J, Prié D, Rothenbuhler A, et al. Therapeutic management of hypophosphatemic rickets from infancy to adulthood. Endocrine Connections 20143 R13-R30. (doi:10.1530/EC-13-0103)
4 Al Kaissi A, Farr S, Ganger R, Klaushofer K \& Grill F. Windswept lower limb deformities in patients with hypophosphataemic rickets. Swiss Medical Weekly 2013143 w13904. (doi:10.4414/smw.2013.13904)

5 Choi IH, Kim JK, Chung CY, Cho T-J, Lee SH, Suh SW, Whang KS, Park HW \& Song KS. Deformity correction of knee and leg lengthening by Ilizarov method in hypophosphatemic rickets: outcomes and significance of serum phosphate level. Journal of Pediatric Orthopaedics 200222 626-631. (doi:10.1097/00004694200209000-00011)

6 Petje G, Meizer R, Radler C, Aigner N \& Grill F. Deformity correction in children with hereditary hypophosphatemic rickets. Clinical Orthopaedics 2008466 3078-3085. (doi:10.1007/s11999008-0547-2)

7 Rohmiller MT, Tylkowski C, Kriss VM \& Mier RJ. The effect of osteotomy on bowing and height in children with X-linked hypophosphatemia. Journal of Pediatric Orthopaedics 199919 114-118.

8 Sharkey MS, Grunseich K \& Carpenter TO. Contemporary medical and surgical management of X-linked hypophosphatemic rickets. Journal of the American Academy of Orthopaedic Surgeons 201523 433-442. (doi:10.5435/JAAOS-D-14-00082)

9 Carpenter TO, Imel EA, Holm IA, Jan de Beur SM \& Insogna KL. A clinician's guide to X-linked hypophosphatemia. Journal of Bone and Mineral Research 201126 1381-1388. (doi:10.1002/jbmr.340)

10 Mäkitie O, Doria A, Kooh SW, Cole WG, Daneman A \& Sochett E. Early treatment improves growth and biochemical and radiographic outcome in X-linked hypophosphatemic rickets. Journal of Clinical Endocrinology and Metabolism 200388 3591-3597. (doi:10.1210/ jc.2003-030036)

11 Quinlan C, Guegan K, Offiah A, Neill RO, Hiorns MP, Ellard S, Bockenhauer D, Hoff WV \& Waters AM. Growth in PHEX-associated $\mathrm{X}$-linked hypophosphatemic rickets: the importance of early treatment. Pediatric Nephrology 201227 581-588. (doi:10.1007/ s00467-011-2046-z)

12 Eralp L, Kocaoglu M, Toker B, Balcı HI \& Awad A. Comparison of fixator-assisted nailing versus circular external fixator for bone realignment of lower extremity angular deformities in rickets disease. Archives of Orthopaedic and Trauma Surgery 2011131 581-589. (doi:10.1007/s00402-010-1162-8)

13 Song H-R, Soma Raju VVJ, Kumar S, Lee S-H, Suh S-W, Kim J$\mathrm{R} \&$ Hong JS. Deformity correction by external fixation and/ or intramedullary nailing in hypophosphatemic rickets. Acta Orthopaedica 200677 307-314. (doi:10.1080/17453670610046073)

14 Eyres KS, Brown J \& Douglas DL. Osteotomy and intramedullary nailing for the correction of progressive deformity in vitamin D-resistant hypophosphataemic rickets. Journal of the Royal College of Surgeons of Edinburgh 199338 50-54.

15 Popkov A, Aranovich A \& Popkov D. Results of deformity correction in children with X-linked hereditary hypophosphatemic rickets by external fixation or combined technique. International Orthopaedics 201539 2423-2431. (doi:10.1007/s00264-015-2814-7)

16 Novais E \& Stevens PM. Hypophosphatemic rickets: the role of hemiepiphysiodesis. Journal of Pediatric Orthopaedics 200626 238-244. (doi:10.1097/01.bpo.0000218531.66856.b7)

17 Fucentese SF, Neuhaus TJ, Ramseier LE \& Ulrich Exner G. Metabolic and orthopedic management of X-linked vitamin D-resistant hypophosphatemic rickets. Journal of Children's Orthopaedics 20082 285-291. (doi:10.1007/s11832-008-0118-9)

18 Rubinovitch M, Said SE, Glorieux FH, Cruess RL \& Rogala E. Principles and results of corrective lower limb osteotomies for patients with vitamin D-resistant hypophosphatemic rickets. Clinical Orthopaedics 1988237 264-270. (doi:10.1097/00003086198812000-00038)

19 Evans GA, Arulanantham K \& Gage JR. Primary hypophosphatemic rickets effect of oral phosphate and vitamin D on growth and surgical treatment. Journal of Bone and Joint Surgery American 198062 1130-1138. (doi:10.2106/00004623-198062070-00010)

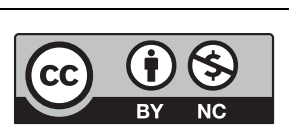

This work is licensed under a Creative Commons Attribution-NonCommercial 4.0 International License. 
20 Berndt M, Ehrich JH, Lazovic D, Zimmermann J, Hillmann G, Kayser C, Prokop M, Schirg E, Siegert B, Wolff G, et al. Clinical course of hypophosphatemic rickets in 23 adults. Clinical Nephrology 199645 33-41.

21 Capelli S, Donghi V, Maruca K, Vezzoli G, Corbetta S, Brandi ML, Mora S \& Weber G. Clinical and molecular heterogeneity in a large series of patients with hypophosphatemic rickets. Bone 201579 143-149. (doi:10.1016/j.bone.2015.05.040)

22 Verge CF, Lam A, Simpson JM, Cowell CT, Howard NJ \& Silink

M. Effects of therapy in X-linked hypophosphatemic rickets. New England Journal of Medicine 1991325 1843-1848. (doi:10.1056/ NEJM199112263252604)

Received in final form 4 September 2017

Accepted 7 September 2017
This work is licensed under a Creative Commons Attribution-NonCommercial 4.0 International License. 